\title{
Diaphragmatic smears are not of additional benefit in the detection of peritoneal spread in gynecological cancers
}

\author{
CELINE MONTAVON $^{1 *}$, UZMA MIRZA $^{1 *}$, ANDRE FEDIER $^{2}$, ANDREAS SCHOETZAU $^{2}$, \\ ROSANNA ZANETTI DÄLLENBACH ${ }^{1}$ and VIOLA HEINZELMANN-SCHWARZ ${ }^{1,2}$
}

\author{
${ }^{1}$ Department of Gynecology and Gynecological Oncology, Hospital for Women; ${ }^{2}$ Ovarian Cancer Research, \\ Department of Biomedicine, University Hospital Basel, University of Basel, CH-4031 Basel, Switzerland
}

Received October 10, 2017; Accepted January 9, 2018

DOI: $10.3892 /$ etm.2018.5957

\begin{abstract}
Peritoneal biopsies (PB) and peritoneal washing (PW) are routine measures in abdominal staging of gynecological malignancies and are used particularly for the assessment of occult microscopic tumor spread to the peritoneal surface including the diaphragm. Cytological diaphragmatic smears (DS) have been suggested as a supplemental tool; however, they are not routinely taken and their usefulness is still unclear. The present study retrospectively evaluated whether DS provide an additional benefit over PB and PW for the detection of peritoneal malignancies in patients with gynecological cancer. The data from patients who underwent laparotomy for suspected gynecological cancer and had DS and either PB, PW or ascites were reviewed. Sensitivity and specificity, and the number upstaged patients were determined. A total of 43 patients were excluded due to benign diagnosis (those with negative DS or PW) and 2 out of the remaining had 2 carcinomas simultaneously. Among these 41 malignancies, DS were positive in $12, \mathrm{PW}$ in 18 and $\mathrm{PB}$ in 19 cases. No case was DS-positive while negative for both PB and PW. Four cases were missed when only PB and 5 when only PW was performed. Notably, no case of peritoneal disease was identified solely on positive DS, indicating that all 23 positive cases (presence of occult peritoneal disease in 56.1\%) were identified by PB and PW together (100\% sensitivity; $62 \%$ specificity). In addition, none of the cases was upstaged solely on positive DS results. Taken together, these data demonstrated that DS do not present an
\end{abstract}

Correspondence to: Professor Viola Heinzelmann-Schwarz, Department of Gynecology and Gynecological Oncology, Hospital for Women, University Hospital Basel, University of Basel, Spitalstrasse 21, CH-4031 Basel, Switzerland

E-mail: viola.heinzelmann@usb.ch

*Contributed equally

Abbreviations: FIGO, International Federation of Gynecology and Obstetrics; NCCN, National Comprehensive Cancer Network

Key words: cytology, diagnosis, diaphragm, ovarian cancer, peritoneal biopsies, upstaging additional benefit to $\mathrm{PW}$ and $\mathrm{PB}$ in the detection of peritoneal gynecological disease.

\section{Introduction}

Surgical staging is an important and critical value to determine the presence of occult disease, the prognosis, and the need for adjuvant chemotherapy in gynecological cancers. In early ovarian cancer (FIGO I/II) proper surgical staging lead to upstaging in one third to one half of these patients $(1,2)$ and is considered as an independent prognostic factor for survival $(3,4)$. In contrast, in more advanced disease (FIGO stage III/IV), where $75 \%$ of patients affected with ovarian cancer present at the time of diagnosis $(5,6)$, cytoreductive surgery is of greater value because the most significant postoperative factor for prognosis is the residual tumor (7).

Today routine measures in abdominal staging of gynecological malignancies include hysterectomy and bilateral salpingo-oophorectomy (if no fertility preservation required), peritoneal washing $(\mathrm{PW})$, peritoneal biopsies $(\mathrm{PB})$, omentum biopsies or omentectomy and lymphonodectomie. PW and PB (including omental biopsies) are routinely employed to assess microscopic tumor spread to the peritoneal surface including the diaphragm.

Intraoperative PW cytology was introduced in 1956 by Keetle and Elkin (8). For ovarian cancer the PW results were included in the FIGO classification system in 1975, and consequently, normal cytologic findings resulted in a significantly increased overall survival regardless of other factors (9). For endometrial cancer, however, the role of PW cytology remained controversy and led to the exclusion of PW cytology in the revised FIGO staging system of 2009: Some studies suggested a prognostic value while others did not $(10,11)$.

Peritoneal smears (PS) and specifically diaphragmatic smears (DS) have been introduced on the basis that the area of examination is larger and this technique thus more sensitive. PS showed sensitivities similar to those of PW but a better specimen quality in one ovarian cancer study (12) but superior to those of PB (97 vs. 59\%) in another (13). On the other hand, DS showed limited reliability: $15 \%$ were unsatisfactory due to too little cellular material or to air-drying artifact, and only 3 of 142 smears were positive for malignant cells (14). 
Although in ovarian cancer the diaphragm is the third most affected organ of occult disease after the peritoneum and the colon (15), PS and in particular DS are not commonly recommended and routinely taken. However, some studies favor a cytology via scrape or diaphragmatic wash, or even blind biopsies even in absence of an obvious macroscopic diaphragmatic disease (16-18), and the National Comprehensive Cancer Network (NCCN) Guidelines for surgical treatment of ovarian cancer suggest blind diaphragmatic biopsies or, alternatively, scraping (19).

Upstaging can occur as a result of presence of occult microscopic disease spread and impacts the treatment and accuracy of risk profile and prognosis $(3,20,21)$, in ovarian and endometrial cancer patients. Owing the lack of respective data, we retrospectively addressed the question as to whether DS provide an additional diagnostic benefit over PW and PB and necessitates upstaging.

\section{Materials and methods}

Study cohort. Files of all patients who underwent laparotomy for suspected gynecological cancer between June 2009 and April 2015 were reviewed using the Gynecological Tumor Database, a computerized oncological database of the Department of Gynecology and Gynecological Oncology, University Hospital Basel, Switzerland. Patients were included from whom DS together with either PB, PW or ascites or all of them have been taken as part as the staging procedure. Women with benign findings were excluded. The study was approved by the local Ethics Committee 'Ethikkomission Nordwest-und Zentralschweiz', EKNZ BASEC 2015-408, in Switzerland in November 2015. Neither written nor verbal informed consent is necessary for this retrospective study.

Sample collection and preparation. Sample collection and preparation was performed the same way for all patients. Upon entering the peritoneal cavity, ascites was aspired. In absence of ascites PW were collected prior to the smears or biopsies by instilling approximately $100-500 \mathrm{ml}$ of warmed-up isotonic saline in the Douglas and paracolic spaces and washing it around. Ascites and PW were collectively referred to as PW and were centrifuged and smears were made in a timely matter at the Department of Pathology, University Hospital Basel.

The DS were always collected by the same surgeon (RZD) using a cervical cytobrush. DS were taken from both the left and right diaphragm, were immediately preserved in a BD SurePath $^{\mathrm{TM}}$ vial, and processed by BD PrepStain ${ }^{\mathrm{TM}}$ (Becton Dickinson AG, Allschwil, Switzerland) according to the manufacturer's protocol. DS and PW smears were stained using the Papanicolaou method.

$\mathrm{PB}$ were usually taken in the upper and lower left and right quadrant, in some cases in the pelvis or Douglas or in the most suspicious areas. PB were fixed in formalin, imbedded into paraffin, and stained with hematoxylin/eosin.

Specimen assessment. All cytological specimens were assessed by two gynecological pathologists at the University Hospital Basel. PB results were considered positive, when at least 1 sample was positive. Positive omental biopsies were also counted PB-positive. DS results were considered positive when at least one out of two samples was positive. Results were negative when DS or PB samples were negative. Sensitivity and specificity were calculated for the comparison of DS vs. PB and PW together (defined as 'gold standard' or reference for evidence of presence of peritoneal disease) to determine the diagnostic value of the measures.

\section{Results}

Patient cohort: Histology and baseline characteristics. A total of 43 patients from whom DS had been taken in order to evaluate occult gynecological disease have been identified in our database between June 2009 and April 2015. Of these, $4(9.3 \%)$ presented with benign findings (3 cystadenomas of the ovary, 1 uterine leiomyoma) and hence were excluded from the study. The remaining 39 patients presented with 41 malignancies: 2 patients had two tumors simultaneously and their results hence counted twice in all calculations (unless a differentiation was made between the two cancers). The 41 malignancies comprised $27(65.8 \%)$ ovarian cancers, 10 (24.4\%) endometrial cancers, $2(4.9 \%)$ primary peritoneal cancers, and $2(4.9 \%)$ 'other' cancers (Table I). The two patients with two simultaneous tumors presented with endometrial and ovarian cancers. The two 'other' cancers were an endometrioid adenocarcinoma of the vaginal stump and a peritoneal cancer originated from the pancreas and both ovaries. The recorded cytological data include cases of ovarian, peritoneal and endometrial cancers. There is no case of cervical cancer, because the cytology is not part of the standard staging procedure. Table I lists the histological subtypes of these cancers.

The baseline characteristics of the patients (mean age and range, Ca-125) and the malignancies (residual disease, nodal status, grading, and International Federation of Gynecology and Obstetrics (FIGO) classification details) are summarized in Table II. Among the 41 malignancies, 18 cases were FIGO stage I/II (43,9\%), 20 were FIGO stage III/IV (48.8\%), and 3 (7.3\%) were of unknown FIGO stage. Table II also summarizes the FIGO stage details for each cancer separately. They show that 11 of the 27 ovarian cancer cases $(40.8 \%)$ were FIGO stage I/II, 14 (51.8\%) were FIGO stage III/IV, and 2 were of unknown FIGO stage. Among the 10 endometrial cancers one half was FIGO stage I/II and the other FIGO stage III/IV, and one peritoneal cancer was FIGO stage I and the other of unknown FIGO stage.

Relationship between the cytological/histopathological results and the FIGO stage. We assigned the cytological/histopathological results (positive or negative findings) for each of the three diagnostic measures (DS, PW/ascites, and PB) to the respective FIGO stages for all 41 malignancies (Fig. 1A) and for each cancer (ovarian, endometrial, peritoneal, and 'other') separately (Fig. 1B). The following data were obtained and presented in more detail for each of the three diagnostic measures.

PW/ascites: Either PW cytology or ascites cytology (collectively referred to as PW) was available in 40 of 41 cases, whereof 18 (45\%) were positive, i.e., contained malignant cells in the abdominal fluid either through positive PW $(8 / 28)$ or through positive ascites (10/12). Three of these 18 positive cases (16.7\%) were FIGO I/II and 13 (72.2\%) were FIGO III/IV. The 
Table I. Histology of the patient cohort.

\begin{tabular}{lc}
\hline Histology & Number of cases $(\mathrm{n}=41)$ \\
\hline Ovarian cancer & 27 \\
Serous & 9 \\
Endometrioid & 5 \\
Mucinous & 4 \\
Clear cell & 1 \\
Borderline & 3 \\
Other & 5 \\
Endometrial cancer & 10 \\
Endometrioid & 5 \\
Serous-papillary & 2 \\
Clear cell & 1 \\
Other & 2 \\
Peritoneal cancer & 2 \\
Endometrioid & 1 \\
Serous & 1 \\
Other & 2 \\
\hline
\end{tabular}

majority $(14 / 18 ; 77.8 \%)$ were ovarian cancers, 2 were endometrial cancer, and 2 peritoneal cancers.

PB: PB were available in 40 cases, where of $19(47.5 \%)$ were positive and were, apart from one unknown case, FIGO III/IV. Four cases were solely positive through positive omentum biopsies. All 21 negative cases were negative through at least 2 PB except for 2 cases, in which only the omentum was biopsied and negative. Positive cases subdivided into 14 ovarian (73.7\%), 1 peritoneal (5.3\%), and 4 (21\%) other cancers.

DS: DS from all 41 cases were available, taken from each the left and the right hemidiaphragm (82 DS samples in total). Positive DS were found in 12 cases (10 ovarian cancers, 1 peritoneal, and 1 'other'): 10 cases with positivity on either side of the diaphragm and 2 cases with positivity only on one side. Nine of the 12 (75\%) positive cases were FIGO III/IV, whereof 6 cases had positive ascites cytology. One case with positive DS was FIGO II (serous ovarian borderline tumor) and 2 positive cases were of unknown FIGO stage. One out of the 29 negative cases was negative owing unsatisfactory amount of material. Interestingly, in 3 cases the diaphragm was clinically involved: 2 showed positive and 1 negative DS. The latter was obviously a false negative case, because the right diaphragm was highly suspicious and the removed adjacent peritoneum was histologically positive. She was a patient with primary debulking by advanced endometrial cancer (FIGO IIIB). In that same case the rest of $\mathrm{PB}$ and $\mathrm{PW}$ was positive.

Detection of peritoneal disease by $P B, P W$, and $D S$. Our data show that among the 41 peritoneal diseases 19 were identified by PB, 18 by PW, and 12 by DS. In order to evaluate whether taking DS in addition to PW and PB is of any benefit in identifying peritoneal disease, i.e., identifies occult diseases not detected by PW and PB, a Venn diagram was calculated (Fig. 2). This diagram presents the number of positive findings for each intersection of the three diagnostic measures
$\mathrm{DS}, \mathrm{PB}$, and $\mathrm{PW}$. This is to determine which measure alone or combination of measures identifies the presence of peritoneal disease and in particular whether one specific measure such as DS identifies a disease that is missed by either one or the other or both other measures. The Venn diagram again displays the positive results for DS (12, red circle), PB (18, blue circle), and PW (19, green circle). The most important finding is that no single case of peritoneal disease was identified solely based on positive DS result (red shaded area), i.e., when both $\mathrm{PB}$ and PW were negative. In addition, 9 PB-positive and 7 PW-positive cases were DS-negative (5 of these DS-negative cases are both PB- and PW-positive), 9 cases were positive for all three measures, 2 DS-positive cases were also PW-positive but PB-negative, and 1 DS-positive cases was PW-negative but PB-positive. Interestingly, $4(2+2)$ positive cases $(17.4 \%)$ were missed based solely on positive PB results and $5(1+4)$ positive cases (21.7\%) solely on positive PW results. Only 9 of $23(39.1 \%)$ positive cases were positive in all three measures.

Taken together, these data demonstrate that all 23 positive cases were detected by PB and PW together with a sensitivity of $100 \%$ and specificity of $62 \%$, indicating that additional DS are not of benefit and hence not useful.

$D S$ and upstaging. We evaluated whether patients were upstaged based on PW and particular on DS. In none of the cases an upstaging was indicated solely based on positive DS. One patient with left-sided ovarian cancer was upstaged from FIGO IA to IC owing of positive PW and possible intraoperative rupturing of the ovarian capsule.

\section{Discussion}

The present retrospective study addressed the question as to whether DS provide an additional benefit in detecting peritoneal disease that otherwise would be missed by the today routine measures (PB and PW/ascites) in abdominal staging. Our results show that i) no single case of peritoneal disease was identified solely based on positive DS results, i.e., all cases were detected by the combination of PB and PW/ascites with $100 \%$ sensitivity, and that ii) no case of upstaging was indicated on this basis. We may conclude that DS is not of any additional (to PB and PW) benefit in diagnosing peritoneal spread in gynecological cancers.

The first major finding of this study is that positive DS results did not reveal peritoneal diseases left undetected by PB and PW, meaning that these two measures together detected all positive cases of peritoneal disease and that hence additional DS were not of additional diagnostic value. This is largely consistent with an earlier study (14) that evaluated the utility of DS as a diagnostic measure and considered it limited: DS were occasionally of insufficient quality and of low specimen yield, and identified only few as positive cases. Unfortunately the study also did not report on direct comparison between DS and PW, leaving open whether the number of positive DS cases was perhaps underestimated. Owing the scarcity and the inconclusiveness of data, DS like PS are not commonly recommended and routinely performed in most institutions.

However, cytology via scrape or diaphragmatic wash or even blind biopsies (16-18) are recommended when macroscopic diaphragmatic disease is not obvious. Blind diaphragmatic 
Table II. Baseline characteristics of the patient cohort.

\begin{tabular}{|c|c|c|c|c|c|}
\hline Characteristics & All $(n=41)$ & Ovarian $(n=27)$ & Endometrial $(n=10)$ & Peritoneal $(n=2)$ & Other $(n=2)$ \\
\hline Age (years), mean (range) & $57.0(16-85)$ & $56.3(16-85)$ & $56.9(38-75)$ & 67.5 & 52.0 \\
\hline $\mathrm{Ca}-125(\mathrm{U} / \mathrm{ml})$ & 626.3 & 658 & 580.7 & 330 & $\mathrm{n} / \mathrm{a}$ \\
\hline \multicolumn{6}{|l|}{ Residual disease } \\
\hline R 0 & 31 & 17 & 7 & 2 & 1 \\
\hline $\mathrm{R} 0-1 \mathrm{~cm}$ & 2 & 1 & 0 & - & - \\
\hline $\mathrm{R}>1 \mathrm{~cm}$ & 1 & 4 & 1 & - & - \\
\hline R X & 7 & 4 & 2 & - & 1 \\
\hline \multicolumn{6}{|l|}{ Nodal status } \\
\hline NO & 25 & 18 & 6 & - & 1 \\
\hline N1 & 6 & 5 & 1 & - & - \\
\hline NX & 10 & 4 & 3 & 2 & 1 \\
\hline \multicolumn{6}{|l|}{ Grade } \\
\hline G1 & 5 & 3 & 1 & 1 & \\
\hline G2 & 6 & 3 & 3 & - & - \\
\hline G3 & 16 & 11 & 5 & - & - \\
\hline G unknown & 14 & 10 & 2 & 1 & 1 \\
\hline FIGO I & $13(31.7 \%)$ & $8(29.7 \%)$ & $4(40 \%)$ & $1(50 \%)$ & \\
\hline FIGO II & $5(12.2 \%)$ & $3(11.1 \%)$ & $1(10 \%)$ & - & $1(50 \%)$ \\
\hline FIGO III & $13(31.6 \%)$ & $9(33.3 \%)$ & $4(40 \%)$ & - & - \\
\hline FIGO IV & $7(17.1 \%)$ & $5(18.5 \%)$ & $1(10 \%)$ & - & $1(50 \%)$ \\
\hline Unknown & $3(7.3 \%)$ & $2(7.4 \%)$ & - & $1(50 \%)$ & - \\
\hline
\end{tabular}

n/a, missing information; -, no cases; FIGO, International Federation of Gynecology and Obstetrics.

biopsies or alternatively diaphragmatic scraping is suggested by NCCN Guidelines for surgical treatment of ovarian cancer (19). Generally, diaphragmatic cytology is of considerable significance. On the one hand, the diaphragm is following the peritoneum and the colon the third most common localization of spread ovarian cancer in almost half (44\%) of the patients (15). The diaphragm is affected in $7 \%$ of the stage I ovarian cancer patients $(1,22)$ and in most cases $(80 \%)$ not only the left but both hemidiaphragms were affected (23), possibly owing the clockwise transportation of peritoneal fluid. On the other hand, an ovarian cancer patient with positive $\mathrm{PW}$ is commonly upstaged from FIGO IA to IC, but will be upstaged according to the new 2013 FIGO classification to FIGO IIIA2 (presence of microscopic peritoneal metastasis beyond the pelvis) in case of a positive diaphragmatic result and given confirmed histological evidence.

Interestingly, a considerable number of positive cases (up to about $20 \%$ ) were missed when only either PB or $\mathrm{PW} /$ ascites was taken. Alike, the combination of either PB or PW/ascites with positive DS results only lowered but not reduced to zero the number of these missed cases, meaning that positive DS results can in particular circumstances, for instance when only either positive $\mathrm{PB}$ or PW/ascites results are available, be helpful in identifying additional positive findings.

The second major finding is that no case upstaged by positive DS results only, suggesting that positive diaphragmatic cytology and in particular DS are not significant and hence are not of benefit in this context. An earlier study, however, showed the utility of diaphragmatic cytology to detect occult metastasis, as $6.5 \%$ of stage I ovarian cancer patients were upstaged to IIIA based on positive diaphragmatic cytology (24).

Upstaging as a result of presence of occult microscopic abdominal disease routinely detected either by biopsies from the omentum, diaphragm, and random sites in the peritoneum or in PW is common and according to International Guideline and has an impact on adjuvant treatment, chemotherapy type to be selected, and accuracy of risk profile and prognosis $(3,20,21)$. Complete and accurate surgical staging and adherence to the guideline has indeed been shown to improve survival outcome in early ovarian cancer (25). On the other hand, incomplete and inaccurate staging, insufficient specimen quality, and guideline incompliance may limit the value of staging as diagnostic and prognostic measures $(1,20,26)$ and may lead to inadequate treatment decisions despite accurate upstaging (27).

The utility regarding the prognostic significance of PW in gynecological neoplasms is also controversial. PW cytology only poorly detected peritoneal implants and predicted clinical outcome analysis of ovarian serous tumors of low malignant potential in one study (28) but was considered as a useful procedure for staging malignant genital tract neoplasms in another (29). A recent retrospective study evaluating the utility of cytology in tumor staging in ovarian and fallopian 
A

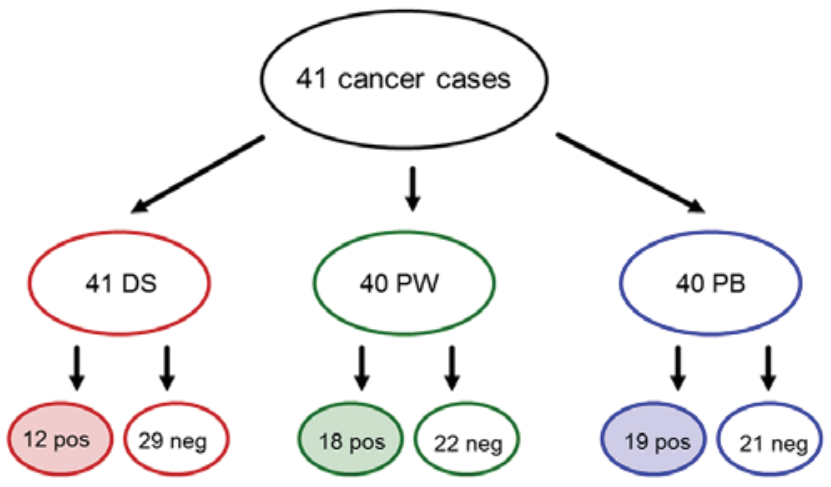

B

\begin{tabular}{|lllllll|}
\hline FIGO & DS + & DS - & PW + & PW - & PB + & PB - \\
\hline Total & $\mathbf{1 2}$ & $\mathbf{2 9}$ & $\mathbf{1 8}$ & $\mathbf{2 2}$ & $\mathbf{1 9}$ & $\mathbf{2 1}$ \\
\hline I & 0 & 13 & 2 & 11 & 0 & 12 \\
\hline II & 1 & 4 & 1 & 4 & 0 & 5 \\
\hline III & 4 & 9 & 7 & 5 & 11 & 2 \\
\hline IV & 5 & 2 & 6 & 1 & 7 & 0 \\
\hline Unknown & 2 & 1 & 2 & 1 & 1 & 2 \\
\hline & & & & & & \\
\hline Cancer & & & & & 14 & \\
\hline Ovarian & 10 & & 14 & & 0 & \\
\hline Endometrial & 0 & & 2 & & 1 & \\
\hline Perintoneal & 1 & & 2 & & & \\
\hline Other & 1 & & 0 & & & \\
\hline
\end{tabular}

Figure 1. (A) Cytological and histopathological results for DS, PW/ascites (collectively PW), and PB showing the positive or negative results for the presence of peritoneal disease. (B) Distribution of the results among the FIGO stages for all malignancies and among each type of cancer (ovarian, endometrial, peritoneal and 'other'). Positive results are shaded. PB, peritoneal biopsies; PW, peritoneal washing; DS, diaphragmatic smears; FIGO, International Federation of Gynecology and Obstetrics; pos/+, positive; neg/-, negative.

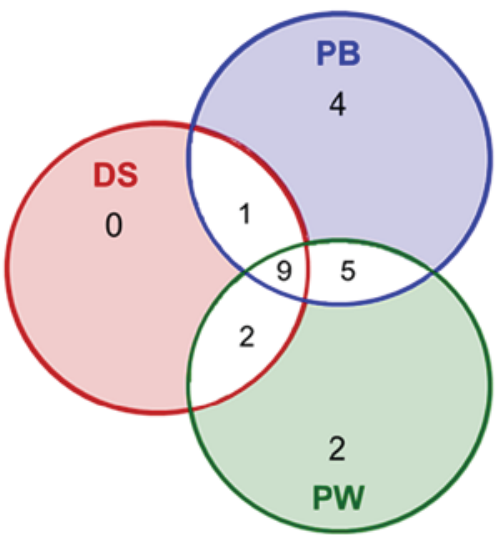

Figure 2. Venn diagram showing the number of positive results for each intersection of the three diagnostic measures DS, PB, and PW/ascites (collectively PW). No single case of peritoneal disease was identified solely based on a positive DS result (red shaded area); thus, all 23 positive cases $(56.1 \%$ of all cases) were identified by PB and PW combined. Cases identified solely by positive $\mathrm{PB}$ are shaded blue and positive PW results are shaded green. A total of 9 positive cases were identified by all three measures. PB, peritoneal biopsies; PW, peritoneal washing; DS, diaphragmatic smears.

tube neoplasms (30) reported upstaging based on positive PW results in $20 \%$ of the patients.

In our study, 5 (out of 23) positive cases were missed based on solely positive PW results. In contrast, positive PW results led to an upstaging in three cases (out of 18: 16.7\%) but not to changes in postoperative treatment. This is consistent with two previous studies reporting $85 \%$ sensitivity and $95 \%$ specificity (31) and $87 \%$ sensitivity and $79 \%$ specificity (32) for the detection of malignant cells in the peritoneal cavity in In FIGO stage I and II ovarian cancer. One of these three patients had left-sided ovarian cancer and was upstaged from FIGO IA to IC, but it was unclear whether upstaging occurred as a consequence of intraoperative rupturing of the ovarian capsule.

Likewise, the value of PW cytology in endometrial cancer also remains ambiguous. Positive PW and poor survival were associated in a 1971 study (9) and positive PW cytology reported to be an independent risk factor for disease to spread to lymph nodes in endometrial cancer in a 2013 study (33). Conversely, contradicting data and low positive cytology rates were reported in other studies (34-38). This led in 2009 to the exclusion of PW-positivity as criterion in the FIGO classification system (39), although taking PW cytology still remains usual in the clinical practice. In our study, only 2 positive PW, 4 positive PB results, and no case of diaphragmatic involvement were found in endometrial cancer.

The occasionally insufficient quality of DS reported in other studies was, apart from one inconclusive result due to poor amount of specimen, not observed in our study. Likewise, $\mathrm{PB}$ and $\mathrm{PW}$ were qualitatively unproblematic and did not deliver inconclusive results, a fact owing to an experienced team including a gynecological oncological surgeon and trained pathologist.

In summary, DS is not of any additional benefit for detection of occult peritoneal disease in ovarian and endometrial cancers and hence is not recommended as a routine measure in clinical practice. 


\section{References}

1. Young RC, Decker DG, Wharton JT, Piver MS, Sindelar WF, Edwards BK and Smith JP: Staging laparotomy in early ovarian cancer. JAMA 250: 3072-3076, 1983.

2. Grabowski JP, Harter P, Buhrmann C, Lorenz D, Hils R, Kommoss S, Traut A and du Bois A: Re-operation outcome in patients referred to a gynecologic oncology center with presumed ovarian cancer FIGO I-IIIA after sub-standard initial surgery. Surg Oncol 21: 31-35, 2012.

3. Trimbos JB, Vergote I, Bolis G, Vermorken JB, Mangioni C, Madronal C, Franchi M, Tateo S, Zanetta G, Scarfone $\mathrm{G}$, et al: Impact of adjuvant chemotherapy and surgical staging in early-stage ovarian carcinoma: European organisation for research and treatment of cancer-adjuvant chemotherapy in ovarian neoplasm trial. J Natl Cancer Inst 95: 113-125, 2003.

4. Timmers PJ, Zwinderman AH, Coens C, Vergote I and Trimbos JB: Understanding the problem of inadequately staging early ovarian cancer. Eur J Cancer 46: 880-884, 2010.

5. Ferlay J, Autier P, Boniol M, Heanue M, Colombet M and Boyle P: Estimates of the cancer incidence and mortality in Europe in 2006. Ann Oncol 18: 581-592, 2007.

6. Benedet JL, Bender H, Jones H III, Ngan HY and Pecorelli S: FIGO staging classifications and clinical practice guidelines in the management of gynecologic cancers. FIGO committee on gynecologic oncology. Int J Gynaecol Obstet 70: 209-262, 2000.

7. du Bois A, Reuss A, Pujade-Lauraine E, Harter P, Ray-Coquard I and Pfisterer J: Role of surgical outcome as prognostic factor in advanced epithelial ovarian cancer: A combined exploratory analysis of 3 prospectively randomized phase 3 multicenter trials: By the Arbeitsgemeinschaft Gynaekologische Onkologie Studiengruppe Ovarialkarzinom (AGO-OVAR) and the Groupe d'Investigateurs Nationaux Pour les Etudes des Cancers de l'Ovaire (GINECO). Cancer 115: 1234-1244, 2009.

8. Keetle WC and Elkin HB: Experience with radioactive colloidal gold in the treatment of ovarian carcinoma. Am J Obstet Gynecol 71: 553-568, 1956.

9. Creasman WT and Rutlidge F: The prognostic value peritoneal cytology in gynecologic malignant disease. Am J Obstet Gynecol 110: 773-781, 1971 .

10. Garg G, Gao F, Wright JD, Hagemann AR, Mutch DG and Powell MA: Positive peritoneal cytology is an independent risk-factor in early stage endometrial cancer. Gynecol Oncol 128: $77-82,2013$.

11. Fadare O, Mariappan MR, Hileeto D, Wang S, McAlpine JN and Rimm DL: Upstaging based solely on positive peritonea washing does not affect outcome in endometrial cancer. Mod Pathol 18: 673-680, 2005.

12. Luesley DM, Williams DR, Ward K, Redman CR and Lawton FG: Prospective comparative cytologic study of direct peritoneal smears and lavage fluids in patients with epithelial ovarian cancer and benign gynecologic disease. Acta Cytol 34 539-544, 1990

13. Jadhon ME, Morgan MA, Kelsten ML, Carlson JA Jr and Mikuta JJ: Cytologic smears of peritoneal surfaces as a sampling technique in epithelial ovarian carcinoma. Obstet Gynecol 75: 102-105, 1990.

14. Jacques SM and Selvaggi SM: Multiple peritoneal cytologies collected during laparotomy for gynecologic malignancy. Diagn Cytopthol 7: 482-486, 1991.

15. Sehouli J, Senyuva F, Fotopoulou C, Neumann U, Denkert C, Werner L and Gülten OO: Intra-Abdominal tumor dissemination pattern and surgical outcome in 214 Patients with primary ovarian cancer. J Surg Oncol 99: 424-427, 2009.

16. Soper JT: Management of early-stage epithelial ovarian cancer. Clin Obstet Gynecol 37: 423-438, 1994.

17. Ozols RF, Rubin SC and Thomas G: Epithelial ovarian cancer. In Hoskins WJ, Perez CA, Young RC, editors. Principles and practice of gynecologic oncology. 4th ed. Philadelphia: Lippincott \& Wilkins: 895-988, 2005.

18. Wheeless CR Jr and Roenneburg ML: Staging of Gynecologic Oncology Patients With Exploratory Laparotomy. In: Atlas of pelvic surgery. On-Line Edition. http://www.atlasofpelvicsurgery.com/10MalignantDisease/1StagingofGynecologicOncolog yPatientsWithExploratoryLaparotomy/cha10sec1.html.

19. NCCN: Epithelial Ovarian Cancer (including Fallopian Tube Cancer and Primary Peritoneal Cancer). Version 2.2013. https:// www.ncen.org/.
20. Lee JY, Kim HS, Chung HH, Kim JW, Park NH and Song YS: The role of omentectomy and random peritoneal biopsies as part of comprehensive surgical staging in apparent early-stage epithelial ovarian cancer. Ann Surg Oncol 21: 2762-2766, 2014.

21. Paik ES, Lee YY, Lee EJ, Choi CH, Kim TJ, Lee JW, Bae DS and Kim BG: Survival analysis of revised 2013 FIGO staging classification of epithelial ovarian cancer and comparison with previous FIGO staging classification. Obstet Gynecol Sci 58: 124-134, 2015.

22. Piver MS, Barlow JJ and Lele SB: Incidence of subclinical metastases in stage I and II ovarian carcinoma. Obstet Gynecol 52: 100-104, 1978.

23. Einenkel J, Ott R, Handzel R, Braumann DU and Horn LC: Characteristics and management of diaphragm involvement in patients with primary advanced stage ovarian, fallopian tube, or peritoneal cancer. Int J Gynecol Cancer 19: 1288-1297, 2009.

24. Eltabbakh GH and Mount SL: Comparison of diaphragmatic wash and scrape specimens in staging of women with ovarian cancer. Gynecol Oncol 81: 461-465, 2001.

25. Lee JY, Kim TH, Suh DH, Kim JW, Kim HS, Chung HH, Park NH, Song YS and Kang SB: Impact of guideline adherence on patient outcomes in early-stage epithelial ovarian cancer. Eur J Surg Oncol 41: 585-591,2015.

26. Buchsbaum HJ and Lifshitz S: Staging and surgical evaluation of ovarian cancer. Semin Oncol 11: 227-237, 1984

27. Powless CA, Bakkum-Gamez JN, Aletti GD and Cliby WA: Random peritoneal biopsies have limited value in staging of apparent early stage epithelial ovarian cancer after thorough exploration. Gynecol Oncol 115: 86-89, 2009.

28. Sneige N, Thomison JB, Malpica A, Gong Y, Ensor J and Silva EG: Peritoneal washing cytologic analysis of ovarian serous tumors of low malignant potential to detect peritoneal implants and predict clinical outcome. Cancer Cytopathol 120: 238-244, 2012.

29. Giordano G, Varotti E, Brigati F and Berretta R: The value of peritoneal washing cytology during intra-abdominal surgery for female genital tract neoplasms. Clin Genitourin Cancer 12: e95-e101, 2014.

30. Davidson W, Madan R, O'Neil M, Tawfik OW and Fan F: Utility of peritoneal washing cytology in staging and prognosis of ovarian and fallopian tube neoplasms: A 10-year retrospective analysis. Ann Diagn Pathol 22: 54-57, 2016.

31. Anastasiadis PG, Romanidis KN, Polichronidis A, Koutlaki NG, Tamiolakis D and Simopoulos K: The contribution of rapid intraoperative cytology to the improvement of ovarian cancer staging. Gynecol Oncol 86: 244-249, 2002

32. Binesh F, Akhavan A, Behniafard N, Zabihi S and Hosseinizadeh E: Prognostic value of peritoneal washing cytology in gynecologic malignancies: A controversial issue. Asian Pac J Cancer Prev 15: 9405-9410, 2014.

33. Garg G, Gao F, Wright JD, Hagemann AR, Zighelboim I, Mutch DG and Powell MA: The risk of lymph-node metastasis with positive peritoneal cytology in endometrial cancer. Int J Gynecol Cancer 23: 90-97, 2013.

34. Kashimura M, Sugihara K, Toki N, Matsuura Y, Kawagoe T, Kamura T, Kaku T, Tsuruchi N, Nakashima H and Sakai H: The significance of peritoneal cytology in uterine cervix and endometrial cancer. Gynecol Oncol 67: 285-290, 1997.

35. Havrilesky LJ, Cragun JM, Calingaert B, Alvarez Secord A, Valea FA, Clarke-Pearson DL, Berchuck A and Soper JT: The prognostic significance of positive peritoneal cytology and adnexal/serosal metastasis in stage IIIA endometrial cancer. Gynecol Oncol 104: 401-405, 2007.

36. Wong FC, Pang CP, Tang SK, Tung SY, Leung TW, Sze WK and Cheung KB: Treatment results of endometrial carcinoma with positive peritoneal washing, adnexal involvement and serosal involvement. Clin Oncol (R Coll Radiol) 16: 350-355, 2004.

37. Zuna RE and Behrens A: Peritoneal washing cytology in gynecologic cancers: Long term follow up of 355 patients. J Natl Cancer Inst 88: 980-987, 1996.

38. Mathew S and Erozan YS: Significance of peritoneal washings in gynecologic oncology. The experience with 901 intraoperative washings at an academic medical center. Arch Pathol Lab Med 121: 604-606, 1997.

39. Lewin SN, Herzog TJ, Barrena Medel NI, Deutsch I, Burke WM, Sun X and Wright J: Comparative performance of the 2009 international Federation of gynecology and obstetrics' staging system for uterine corpus cancer. Obstet Gynecol 116: 1141-1149, 2010.

This work is licensed under a Creative Commons Attribution-NonCommercial-NoDerivatives 4.0 International (CC BY-NC-ND 4.0) License. 